\title{
VECTOR MESON PRODUCTION AT HERA
}

\author{
JAN FIGIEL \\ Institute of Nuclear Physics Cracow, Poland \\ e-mail: Jan.Figiel@ifj.edu.pl \\ on behalf of the ZEUS and H1 collaborations
}

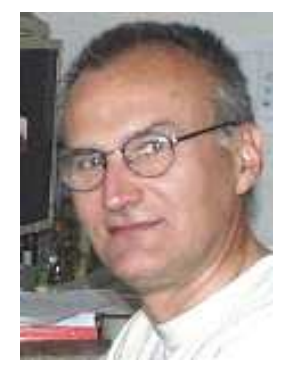
New results on elastic electroproduction and proton-dissociative photoproduction at large $|t|$
of $\rho, \phi$ and $J / \psi$ mesons are presented. They are interpreted within perturbative QCD.

\section{Introduction}

At high energy the diffractive electroproduction of the vector mesons (VM) is a two-stage process: at first the virtual photon fluctuates into VM which then interacts with the target. The latter is the process of interest and in analogy with soft hadron-hadron interactions can be interpreted within Regge phenomenology 1 . However at large $Q^{2}$ or mass of the meson $M_{V}$ (and in principle $|t|)$ the range of the VM-p interaction is small: it is "hard" and perturbative QCD can be applied 2 . It describes the process via exchange of colour singlet system of gluons ( 2 gluons in leading order - Figure 11). Contrary to Regge approach, in pQCD framework the steep rise of

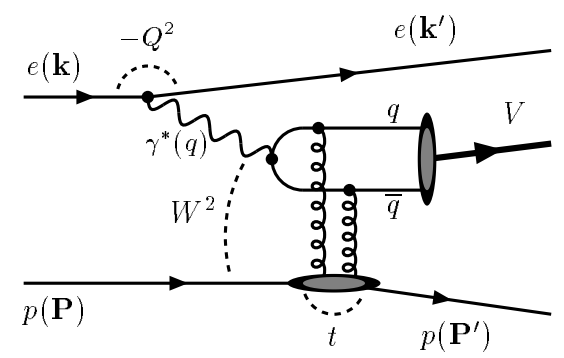

Figure 1: The pQCD diagram for VM electro-production via 2-gluon exchange. $W$ is energy in $\gamma^{*} p$ CMS, $Q^{2}$ is photon virtuality and $t$ denotes square of the 4-momentum transfer between incoming and outgoing proton. 

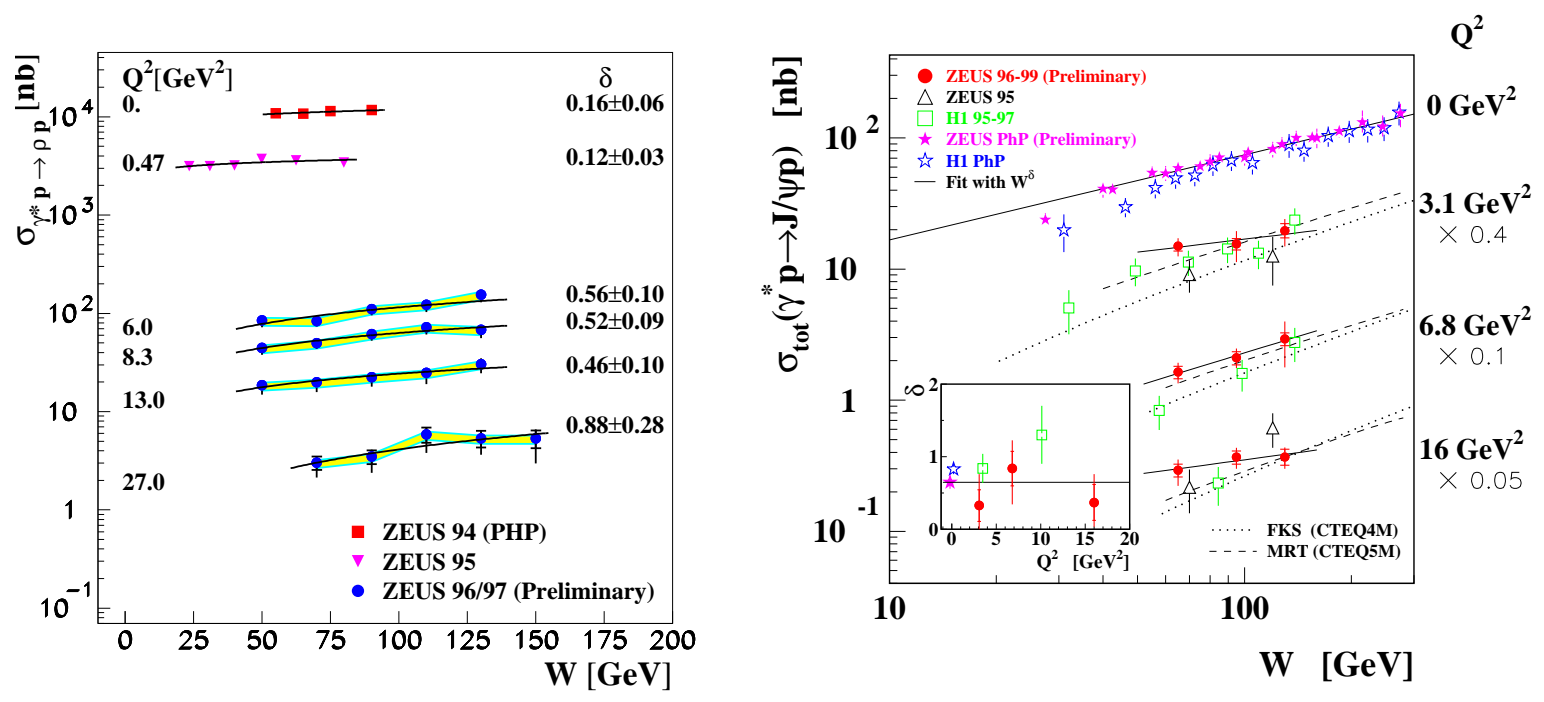

Figure 2: Left - the $\rho$ cross section, right - the $J / \psi$ cross section; solid lines represent fits to the ZEUS data with the formula $\sim W^{\delta}$ and dotted and dashed ones - the predictions of pQCD models (see text). The inset displays the fitted value of $\delta$ as a function of $Q^{2}$.

the VM production cross section and violation of the s-channel helicity conservation (SCHC) is predicted. The transition between soft and hard regime is a test ground of the QCD and is studied intensively at HERA .

\section{Elastic electroproduction of $\rho$ and $J / \psi$ mesons}

Diffractive elastic electroproduction of $\rho$ mesons has been measured by the ZEUS collaboration 3 . The cross section $\sigma_{\gamma^{*} p \rightarrow \rho p}$ is shown in Figure 2 (left) as a function of $W$ in $\operatorname{different} Q^{2}$ intervals. It is parametrised as $\sim W^{\delta}$; magnitude of power $\delta$ reflects steepness of its energy dependence. At $Q^{2} \sim 0 \delta$ is close to 0.2 , value typical for soft hadronic interactions whereas at large $Q^{2}$ it is much bigger indicating strong energy dependence typical for hard colour singlet exchange. Thus large $Q^{2}$ provides "hard" QCD scale. On the right side of Figure 2 the recent measurement of the $J / \psi$ cross section by ZEUS $\mathrm{G}$ and $\mathrm{H} 1 \mathrm{O}$ experiments are shown. As before the energy dependence in $Q^{2}$ intervals is fitted with power law formula. However now one observes steep rise of the cross section with $W(\delta \simeq 0.65)$ irrespectively of $Q^{2}$ value: "large" $M_{\psi}^{2}$ seems to be sufficient hard scale. The pQCD based models 6 , relating the $J / \psi$ cross section to the gluon density in proton reproduce the behaviour of the data.

\section{Photoproduction of $\rho, \phi$ and $J / \psi$ mesons at large $|t|$}

In principle large $|t|$ should provide hard QCD scale however this cannot be tested in elastic VM production where typical values of $|t|$ are lower than $1 \mathrm{GeV}^{2}$. That is why the ZEUS collaboration preformed a dedicated measurement 0 of proton-dissociative photoproduction of vector mesons in the energy range $80<W<120 \mathrm{GeV}$ reaching the value of $|t| \simeq 12 \mathrm{GeV}^{2}$. In this process, $\gamma^{*} p \rightarrow V N$, vector meson and system $N$ of proton dissociation products are separated by large rapidity gap so mechanism of Pomeron (or colour singlet) exchange can be invoked. The differential cross sections $d \sigma / d t$ are presented in Figure 3. Contrary to elastic VM production they do not follow the exponential but rather power law dependence $\sim|t|^{n}$, natural in pQCD framework. The heavier VM the less steep t dependence of the cross section: $n=-3.31 \pm 0.02$ (stat.) \pm 0.12 (syst.) for $\rho, n=-2.77 \pm 0.07$ (stat.) \pm 0.17 (syst.) for $\phi$ and $n=-1.7 \pm 0.2$ (stat.) \pm 0.2 (syst.) for $J / \psi$ meson. In case of $J / \psi$ production the model 8 assuming pQCD "Pomeron" coupling to single parton in the proton describes the data. 
ZEUS
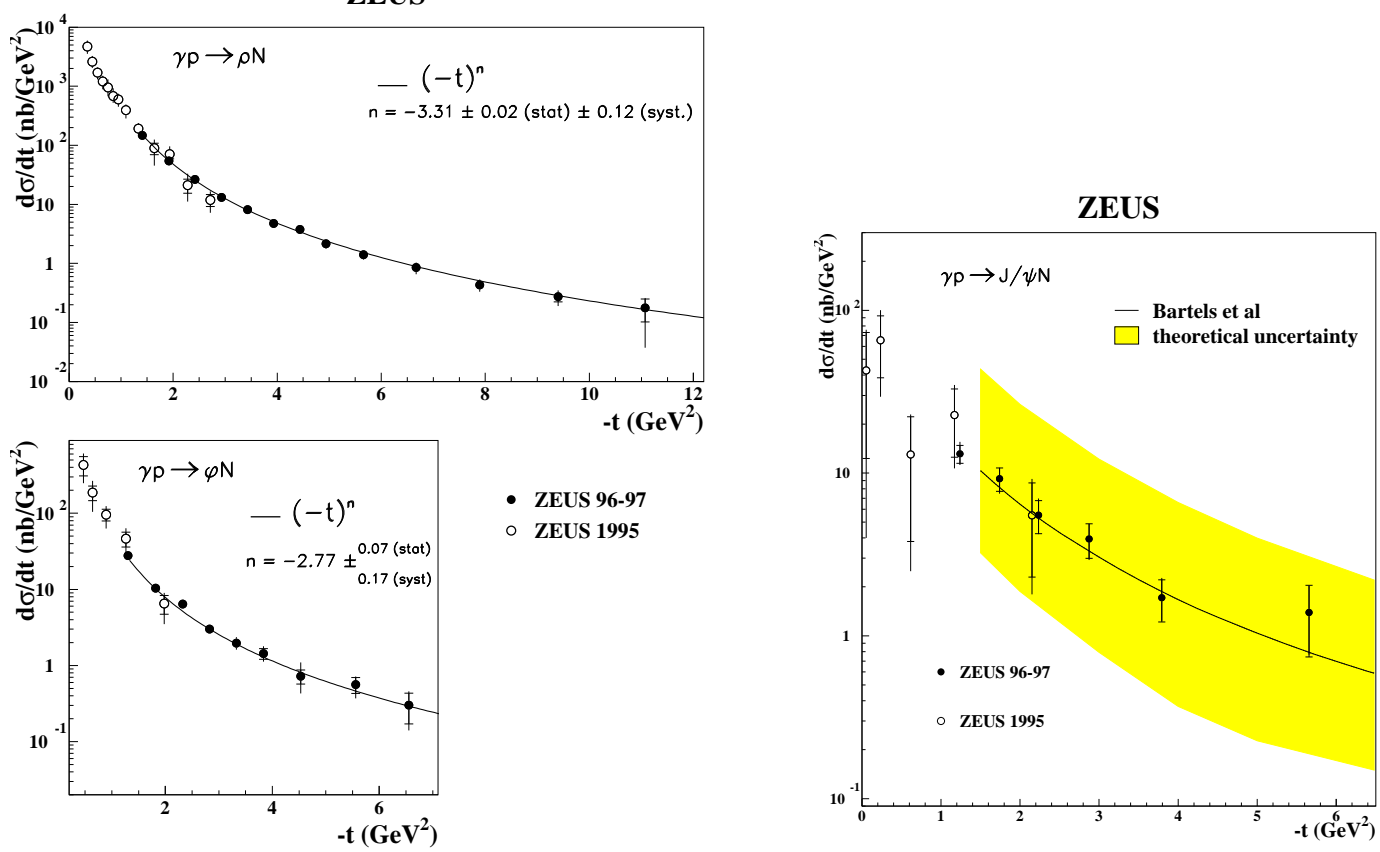

Figure 3: Left up - differential cross section for $\gamma^{*} p \rightarrow \rho N$, left down - $\gamma^{*} p \rightarrow \phi N$, solid lines represent fits with formula $\sim|t|^{n}$. Right $-\gamma^{*} p \rightarrow J / \psi N$, solid line represents prediction of the model described in text.
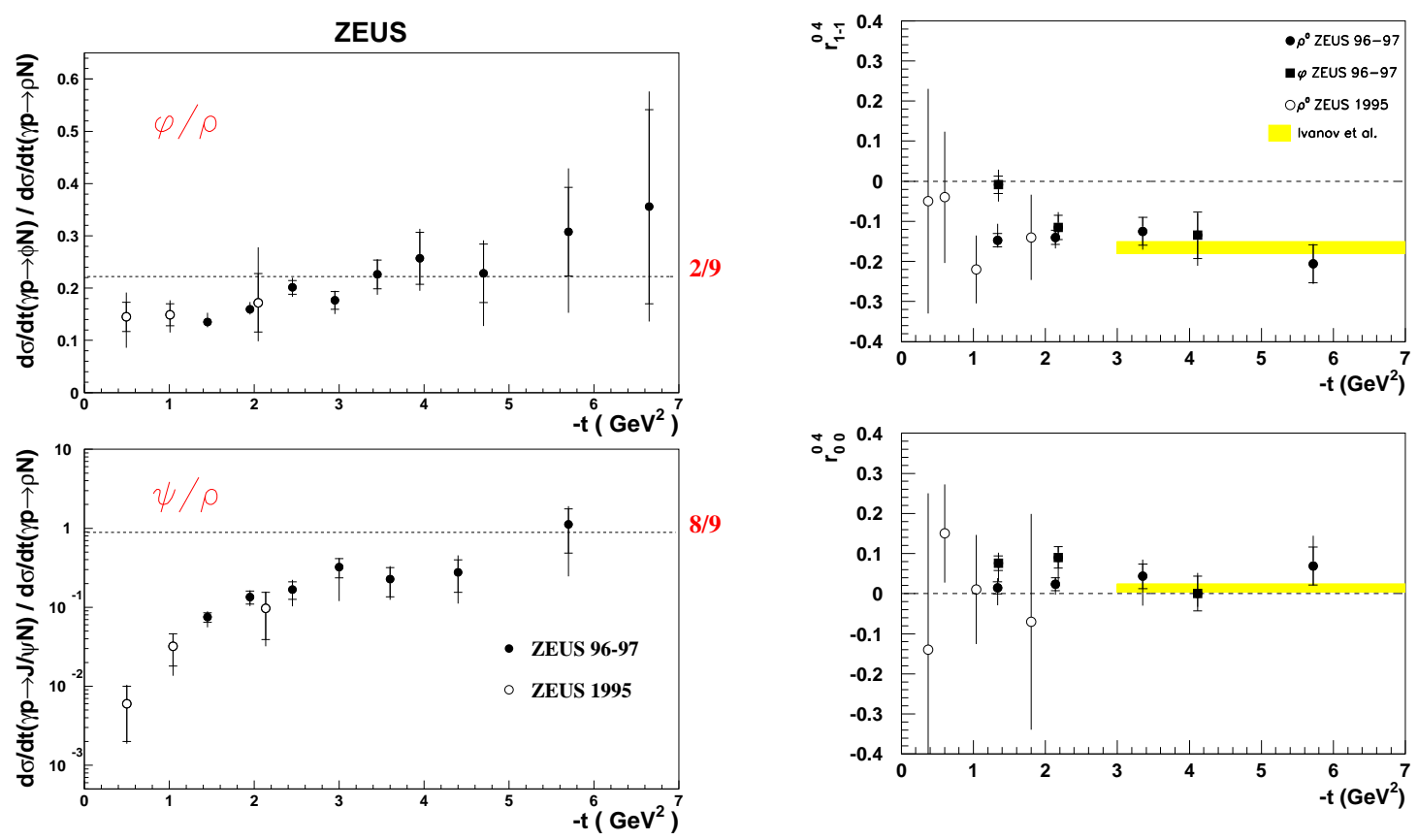

Figure 4: Left - the cross sections ratios $\phi / \rho$ and $\psi / \rho$ in function of $|t|$, right - the spin density matrix elements for $\rho$ and $\phi$ mesons in function of $|t|$, the grey bands represent predictions of the pQCD based model.

The ratios of cross sections $\phi / \rho$ and $\psi / \rho$ are the next subject of interest as their values reflect the underlying mechanism of VM production. In particular if photon couples directly to quarks in VM and assuming flavour independent production mechanism which is natural in "hard" interactions, these ratios are determined by quark composition of vector mesons: $\phi: \psi: \rho=$ $2: 8: 9$. These asymptotic values of the ratios were already observed at larger $Q^{2}$ values 61 . 1 . 
The first measurements of the VM cross section ratios as a function of $|t|$ are presented in Figure 1 (left). Both ratios approach asymptotic values however the $\psi / \rho$ ratio at higher value of $|t|$ than $\phi / \rho$. This general behaviour is consistent with assumption that $|t|$ provides hard scale.

From angular distributions (in s-channel frame) of VM decay products one can determine its spin state which should be the same as photon if helicity is conserved. The s-channel helicity conservation is natural feature of soft hadronic interactions however its smallviolation in

electroproduction of $\rho$ mesons was predicted in $\mathrm{pQCD}$ and observed experimentally $\mathrm{G}$. In photoproduction the nearly real photon is transversely polarised and the same should be polarisation of $\mathrm{VM}$ if SCHC holds. In particular the spin density matrix elements $r_{1-1}^{04}$ and $r_{00}^{04}$, reflecting double and single helicity flip amplitudes respectively, should be zero. These matrix elements are shown in Figure 1 (right) in function of $|t|$. There is clear indication of non-zero double spin flip contribution at $|t|>3 \mathrm{GeV}^{2}$ which is reproduced by pQCD model 10 .

\section{Summary}

During last year quite a lot of data on vector meson production at HERA were released. Their statistics and quality enabled multi-variable analysis yielding ineresting results. In particular the production of heavy mesons shows expected features of a short distance (hard) process. For light mesons the virtuality of the photon controls the transition between soft $\left(Q^{2} \simeq 0\right)$ and hard ("large" $Q^{2}$ ) limits of strong interactions. There is growing experimental evidence that also $|t|$ provides hard scale. Production of vector mesons at HERA turns out to be a playground for non-perturbative and perturbative QCD.

\section{Acknowledgments}

I thank the DESY Directorate for their support which enabled my participation in this very interesting conference. My work was also supported by the Polish State Committee for Scientific Research (KBN), grant No. 2P03B04616.

\section{References}

1. A. Donnachie and P. V. Landshoff, Nucl. Phys. B 244, 322 (1984).

2. M. G. Ryskin, Z. Phys. C 57, 89 (1993), S. J. Brodsky, Phys. Rev. D 50, 3134 (1994).

3. ZEUS collaboration, contributed paper 880 to ICHEP2000, Osaka 2000.

4. ZEUS collaboration, contributed paper 879 to ICHEP2000, Osaka 2000.

5. H1 collaboration, C. Adloff et al., Eur. Phys. J. C 10, 373 (1999).

6. L. Frankfurt, W. Koepf and M. Strikman, , Phys. Rev. D 57, 512 (1998),

A. D. Martin, M. G. Ryskin and T. Teubner, Phys. Rev. D 62, 014022 (2000).

7. ZEUS collaboration, contributed paper 884 to ICHEP2000, Osaka 2000.

8. J. Bartels et al., Phys. Lett. B 375, 301 (1996).

9. H1 collaboration, C. Adloff et al., Eur. Phys. J. C 13, 371 (2000), ZEUS collaboration, J. Breitweg et al., Eur. Phys. J. C 12, 393 (2000).

10. D. Yu. Ivanov et al., Phys. Lett. B 478, 101 (2000).

11. ZEUS collaboration, J. Breitweg et al., Phys. Lett. B 487, 279 (2000). 